\title{
Narrowband selected high-redshift galaxy candidates contaminated by lower redshift [OIII] ultra-strong emitter line galaxies ${ }^{\star}$
}

\author{
Aurélie Pénin ${ }^{1,2}$, Jean-Gabriel Cuby ${ }^{1}$, Benjamin Clément ${ }^{3,6}$, Pascale Hibon ${ }^{5}$, Jean-Paul Kneib ${ }^{1,4}$, \\ Paolo Cassata $^{1}$, and Olivier Ilbert ${ }^{1}$
}

\author{
1 Aix Marseille Université, CNRS, Laboratoire d'Astrophysique de Marseille (LAM), UMR 7326, 13388 Marseille, France \\ e-mail: aurelie.c.penin@gmail.com \\ 2 Astrophysics and Cosmology Research Unit, School of Mathematical Sciences, University of KwaZulu-Natal, \\ 4041 Durban, South Africa \\ 3 Steward Observatory, University of Arizona, 933 North Cherry Avenue, Tucson, AZ 85721, USA \\ ${ }^{4}$ Laboratoire d'Astrophysique, École Polytechnique Fédérale de Lausanne (EPFL), Observatoire de Sauverny, \\ 1290 Versoix, Switzerland \\ 5 Gemini Observatory, Casilla 603, La Serena, Chile \\ ${ }^{6}$ CRAL, Observatoire de Lyon, Université Lyon 1, 9 avenue Ch. André, 69561 Saint Genis Laval Cedex, France
}

Received 20 October 2014 / Accepted 24 February 2015

\begin{abstract}
Context. Lyman-break galaxies (LBG) and narrowband (NB) surveys have been successful at detecting large samples of high-redshift galaxies. Both methods are subject to contamination from low-redshift interlopers.

Aims. Our aim is to investigate the nature of low-redshift interlopers in NB Lyman- $\alpha$ emitters (LAE) searches.

Methods. From previous HAWK-I NB imaging at $z \sim 7.7$, we identify three objects that would have been selected as high-redshift LAEs had our optical data been one magnitude shallower (but still one to two magnitudes fainter than the near infrared data). We follow up these objects in spectroscopy with X-Shooter at the VLT.

Results. Despite low quality data due to bad weather conditions, for each of the three objects we identify one, and only one emission line, in the spectra of the objects that we identify as the [OIII]5007 $\AA$ line. From this result, combined with spectral energy density fitting and tests based on line ratios of several populations of galaxies, we infer that the three objects are ultra-strong line emitters at redshifts $\sim 1.1$.

Conclusions. From this work and the literature we remark that the [OIII] line appears to be a common source of contamination in high-redshift LBG and LAE samples and we suggest that efforts be made to characterize with high accuracy the [OIII] luminosity function out to redshift $\sim 3$ or higher.
\end{abstract}

Key words. galaxies: evolution - galaxies: high-redshift - techniques: imaging spectroscopy

\section{Introduction}

The study of the highest redshift galaxies and quasars at $z>7$ provides insights into the early stages of galaxy formation and evolution and on the ionization state of the inter-galactic medium (IGM). Much progress has been achieved over the past decade in assembling samples of high-redshift galaxies from essentially two techniques: the Lyman-break technique selecting Lyman-break galaxies (LBG) and the narrowband (NB) imaging technique targeting Ly $\alpha$ emitters (LAEs).

The Lyman Break method looks at the Lyman continuum break in the UV spectra of galaxies. Pioneered by Steidel et al. (2003) at redshift $\sim 3$, this technique has been progressively applied to higher redshifts. As redshift increases, the Ly $\alpha$ forest becomes so dense that the continuum blueward of the Ly $\alpha$ line is almost entirely suppressed, in practice becoming the signature used to select high-redshift galaxies with the LBG technique. At

* Based on observations made with ESO telescopes at the La Silla Paranal Observatory under programmes ID 385.A-1025(A) and 181.A-0485. redshift 7 , the break occurs around $1 \mu \mathrm{m}$ and galaxies can only be observed in the near infrared.

In several studies performed after the installation of the WFC3 camera on board HST in 2009, the Lyman-break technique has been used to search for galaxies up to redshifts 10 or higher. Samples of hundreds of galaxies at redshifts 7 to 9 have been assembled, and while their spectroscopic confirmation remains an extremely difficult endeavor because of their faintness, the high-redshift nature of these samples is extremely robust and indisputable, with estimated contamination rates of the order of 10 to $20 \%$. Bouwens et al. (2015) provides a recent and comprehensive compilation of $z \sim 4$ to $z \sim 10$ LBG galaxy samples from various HST data sets. The $z \sim 7$ and $z \sim 8$ samples have approximately 600 and 200 objects, respectively, with an estimated contamination rate of about $10 \%$, which is much lower than the contamination rate estimated for earlier samples (Trenti et al. 2011).

Searching high-redshift LAEs from NB imaging relies on combining a flux excess in the NB filter with a break at the wavelength of the NB filter that corresponds to the break below the Ly $\alpha$ line mentioned above. At least one broadband filter 
overlapping with, or redder than, the NB filter is required to select line emitting objects, and additional redder broadband filters may provide supplemental information on the colours of the objects. The NB filters are usually centred on regions of low $\mathrm{OH}$ emission from the sky for optimum sensitivity, therefore leading to discrete redshift intervals, namely 5.7, 6.5, 7.3, 7.7 and 8.8. This technique has been extremely successful at finding $z \sim 6.5$ LAES (Hu et al. 2004, 2010; Kashikawa et al. 2006; Ouchi et al. 2010; Nakamura et al. 2011; Cassata et al. 2011). These samples have been robustly confirmed in spectroscopy, with estimated contamination rates close to or below $30 \%$. The technique has been used into the near IR to search for even higher redshifts, see e.g. Cuby et al. (2007), Willis et al. (2008) for pioneering searches at redshifts 8.8 with small format near IR detectors. With the advent of large mosaics of near IR arrays, more recent searches have been carried out at redshifts 7.3, 7.7 and 8.8 (Tilvi et al. 2010; Hibon et al. 2010, 2011; Krug et al. 2012; Clément et al. 2012; Shibuya et al. 2012; Milvang-Jensen et al. 2013; Matthee et al. 2014; Konno et al. 2014). Thus far, only one of the LAE candidates from these searches has been confirmed spectroscopically at $z \sim 7$.3. It was found with the Suprime-Cam CCD camera equipped with a special NB filter at the Subaru telescope (Konno et al. 2014).

Both the LBG and the NB observations have now convincingly pointed to a decline of the $\operatorname{Ly} \alpha$ radiation at redshifts $>7$, possibly because of an increasing fraction of neutral hydrogen from $z \sim 6$ when the Universe was completely re-ionized. Evidence for this attenuation of $\operatorname{Ly} \alpha$ radiation comes from the strong decline of the co-moving density of LAEs inferred from NB searches, as well as from the declining fraction beyond $z \sim 6.5$ of LAEs among LBGs (Schenker et al. 2012; Pentericci et al. 2014; Tilvi et al. 2014). From the hundreds of LBGs selected at redshifts $>7$, less than a handful have been spectroscopically confirmed (Vanzella et al. 2011; Finkelstein et al. 2013).

Throughout these intensive searches for high-redshift galaxies, several cases of contamination by low-redshift interlopers or spurious sources have been reported. Contamination of high-redshift LBG and LAE photometric samples can affect the constraints derived from these samples on the properties of high-redshift galaxies or on the neutral fraction of the Universe during the re-ionization epoch. It is expected that contamination increases with redshift, when the objects are fainter and less constrained from their photometry since the object may be detected in one or two bands only. This is exemplified by the spectroscopic identification at a redshift of 2.08 of an LBG candidate at $z \sim 11$ (Hayes et al. 2012) and the likely identification at a redshift of 2.19 of another LBG candidate at $z \sim 12$ (Brammer et al. 2013; Capak et al. 2013). In an independent parallel survey with HST/WFC3, Atek et al. (2011) show that extreme emission line galaxies at $z \sim 1-2$ can mimic the broadband colours of $z \sim 8$ LBGs, the high equivalent width (EW) lines being mistaken for the break at the Ly $\alpha$ line. Similarly, ultra-strong emission line galaxies can contaminate LAE samples by mimicking a colour break on the blue side of the NB filter. Ultra-strong emission line galaxies selected from optical NB data are studied e.g. in Kakazu et al. (2007), while Matthee et al. (2014) and Kochiashvili et al. (2015) are two recent studies of NB-selected objects in the near infrared.

In this paper, we investigate the nature of possible contaminants of high-redshift LAE candidates, using our own NB data at $1.06 \mu \mathrm{m}$. To do so, we use the LAE samples from one of our previous studies (Clément et al. 2012) in which we have selected high EW line emitting objects at low redshift that would have been selected as LAE candidates at $z \sim 7.7$, if the optical observations had been one magnitude shallower. From VLT-X-Shooter spectroscopy, we investigate the nature of these objects.

We use $A B$ magnitudes throughout this paper. We assume a flat $\Lambda$ CDM model with $\Omega_{\mathrm{m}}=0.30$.

We describe the observations in Sect. 2, and investigate the nature of these objects in Sect. 3. Finally, we discuss the implications of our results for high-redshift surveys in Sect. 4.

\section{Data}

\subsection{Photometry}

Clément et al. (2012) present the results of a deep NB search of LAEs at $1.06 \mu \mathrm{m}$ (corresponding to $\operatorname{Ly} \alpha$ at $z \sim 7.7$ ) performed at the VLT with the HAWK-I instrument. This study did not identify $z=7.7$ LAEs, allowing us to infer strong constraints on the evolution of the Ly $\alpha$ luminosity function from redshift 6.5 . The NB survey reached a $3 \sigma$ aperture corrected limiting magnitude of 26.65, while the CFHTLS optical data have limiting magnitudes of 27.4, 28.2, 28.0, 27.4, and 26.6 in the $u^{\star}, g^{\prime}, r^{\prime}, i^{\prime}, z^{\prime}$, respectively.

The NB1060 data were acquired over two epochs separated by one year to prevent the selection of transient object that would be detected in a one-epoch stack and not in the other. To select high-redshift candidates, Clément et al. (2012) applied the following criteria:

1. $N B 1060 \geq 5 \sigma \wedge N B 1060_{\text {epoch } 1} \geq 2 \sigma \wedge N B 1060_{\text {epoch } 2} \geq 2 \sigma$;

2. No detection above the $2 \sigma$ level in any of the visible broadband filter;

3. $2 \leq Y-N B 1060 \leq 2.7$;

4. $N B 1060-J \leq 0$ with $1 \sigma$ significance.

While Criteria 4 prevents the selection of T-dwarfs stars, which usually have $N B 1060-J \geq 0$, Criteria 2 corresponds to the following colour criteria :

$$
\begin{aligned}
& -u_{2 \sigma}^{\star}-N B 1060 \geq 1.7 ; \\
& -g_{2 \sigma}^{\prime}-N B 1060 \geq 2.5 ; \\
& -r_{2 \sigma}^{\prime}-N B 1060 \geq 2.3 ; \\
& -i_{2 \sigma}^{\prime}-N B 1060 \geq 1.8 ; \text { and } \\
& -z_{2 \sigma}^{\prime}-N B 1060 \geq 0.9
\end{aligned}
$$

In the process of selecting high-redshift candidates, seven peculiar objects were identified in the CFHTLS-D4 field. They are moderately bright in the $N B 1060$ filter with $A B \leq 25.3$, with a signal-to-noise ratio $S / N \geq 5 \sigma$ while they have $N B 1060$ $-J<-1.5$ and, in particular, $z-N B 1060>1.8$, typical of highredshift LAEs. Nevertheless, they are detected in optical broadband filters with magnitudes $\geq 27$ in the $u^{\star}, g^{\prime}, r^{\prime}, i^{\prime}$ filters and $\geq 26.5$ in the $z^{\prime}$. Clearly, these strong, line-emitting objects would have been selected as genuine LAE candidates with slightly shallower optical data. We observed the three brightest objects in spectroscopy with X-Shooter to elucidate their nature. The photometric properties of these three objects are reported in Table 1 and thumbnail images in all photometric bands are shown in Fig. 1.

\subsection{The $X$-Shooter observations}

The objects were observed with the X-Shooter instrument (D'Odorico et al. 2006; Vernet et al. 2011) mounted at UT2 of the ESO-VLT on the nights of the 19th and 20th of July 2010. The X-Shooter instrument is a three-arm single object spectrograph covering an extremely wide spectral range from 300 
Table 1. Photometric data of the three NB selected objects we studied.

\begin{tabular}{cccccc}
\hline \hline Instrument & Filter & Central wavelength $(\mathrm{nm})$ & Obj1 & Obj2 & Obj3 \\
\hline WIRCam & $K \mathrm{~s}$ & 2146 & $>24.70$ & $>24.70$ & $>24.70$ \\
WIRCam & $H$ & 1631 & $>24.70$ & $>24.70$ & $>24.70$ \\
HAWK-I & $J$ & 1258 & $>26.53$ & $>26.53$ & $>26.53$ \\
HAWK-I & $N B 1060$ & 1062 & $24.3 \pm 0.1$ & $24.30 \pm 0.06$ & $24.52 \pm 0.05$ \\
Megacam & $z^{\prime}$ & 890 & $>26.6$ & $26.59 \pm 0.40$ & $26.43 \pm 0.31$ \\
Megacam & $i^{\prime}$ & 770 & $27.1 \pm 0.2$ & $>27.43$ & $27.02 \pm 0.19$ \\
Megacam & $r^{\prime}$ & 625 & $27.3 \pm 0.2$ & $27.41 \pm 0.21$ & $>28.0$ \\
Megacam & $g^{\prime}$ & 486 & $27.3 \pm 0.2$ & $27.44 \pm 0.19$ & $27.89 \pm 0.27$ \\
Megacam & $u^{\star}$ & 378 & $27.1 \pm 0.3$ & $>27.37$ & $>27.37$ \\
\hline
\end{tabular}

Notes. The significance of the limiting magnitudes is $3 \sigma$.

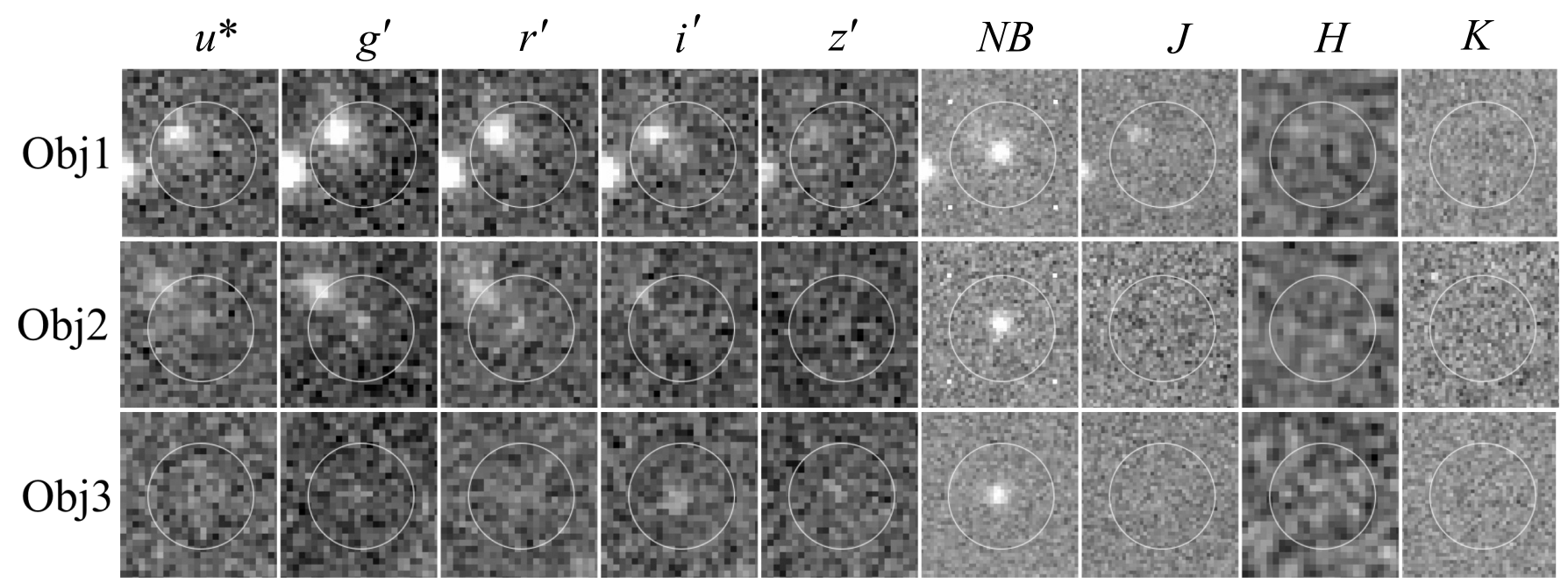

Fig. 1. Thumbnail images of the three objects. The size of the thumbnails is $2.65 \times 2.65 \operatorname{arcsec}^{2}$.

Table 2. Slits and exposure times for each of three X-Shooter arms used for each of the three objects.

\begin{tabular}{|c|c|c|c|c|c|c|}
\hline \multirow[t]{2}{*}{ Id } & \multirow[t]{2}{*}{$\alpha(\mathrm{J} 2000)$} & \multirow[t]{2}{*}{$\delta(\mathrm{J} 2000)$} & \multicolumn{3}{|c|}{ Exposure time (s) } & \multirow[t]{2}{*}{ Slit size } \\
\hline & & & $U V B$ & $V I S$ & NIR & \\
\hline Obj1 & 334.143 & -17.5566 & $3 \times 1700$ & $3 \times 1700$ & $3 \times 1800$ & $1.2^{\prime \prime}$ \\
\hline Obj2 & 334.099 & -17.5326 & $2 \times 1100+600$ & $2 \times 1100+600$ & $3 \times 1200$ & $1.2^{\prime \prime}$ \\
\hline Obj3 & 334.208 & -17.5349 & $3 \times 1750+1287$ & $3 \times 1750+1287$ & $3 \times 1800$ & $1.5^{\prime \prime}$ \\
\hline
\end{tabular}

Notes. Dithering was applied between individual exposures.

to $2500 \mathrm{~nm}$. The spectral resolution of the instrument varies from $R \sim 3300$ to $R=5500$ depending on wavelength for a $1.2^{\prime \prime}$ slit.

The observations took place just after a cold front had passed over Paranal, and as a result the observing conditions were extremely poor, windy, non-photometric, and with poor seeing. The seeing was of the order of $1.5^{\prime \prime}$ in average, and up to $2^{\prime \prime}$. The total time that could be spent on the targets ended up being much lower than originally planned, and different slits were used in a desperate effort to catch up with the seeing conditions. The detailed observation parameters are provided in Table 2 . We observed the three objects three times with dithering between exposures. We achieved a limiting magnitude of $\sim 23.5$ at the wavelength of the $N B 1060$ by measuring the standard deviation over $5 \AA$ and over the whole width of flux calibrated spectra.

We reduced the data using the EsoRex X-Shooter pipeline version 1.3.7, including sky subtraction and removal of bad pixels and cosmic rays. We then stacked the calibrated spectra using different methods: average, median, and average with rejection of outliers. We carried out the following analysis using the combination of the average with rejection of outliers spectra for each object.

\section{Analysis}

We searched emission lines in each of the 2D stacked spectra from a careful visual inspection. Interestingly, we only detect one emission line in each of the three $2 \mathrm{D}$ spectra within the NB1060 filter bandwidth. The reality of these lines was carefully checked by tracking their spatial positions along the slit on the 2D spectrum on each of the three individual dithered images forming the $2 \mathrm{D}$ stacks. For each object, we extract a $1 \mathrm{D}$ line spectrum. Both 1D and 2D spectra for each of the three objects are shown in Fig. 2.

For each object, we evaluated the slit losses when measuring the line fluxes. These slit losses were evaluated considering the (poor) seeing conditions prevailing during the observations, 

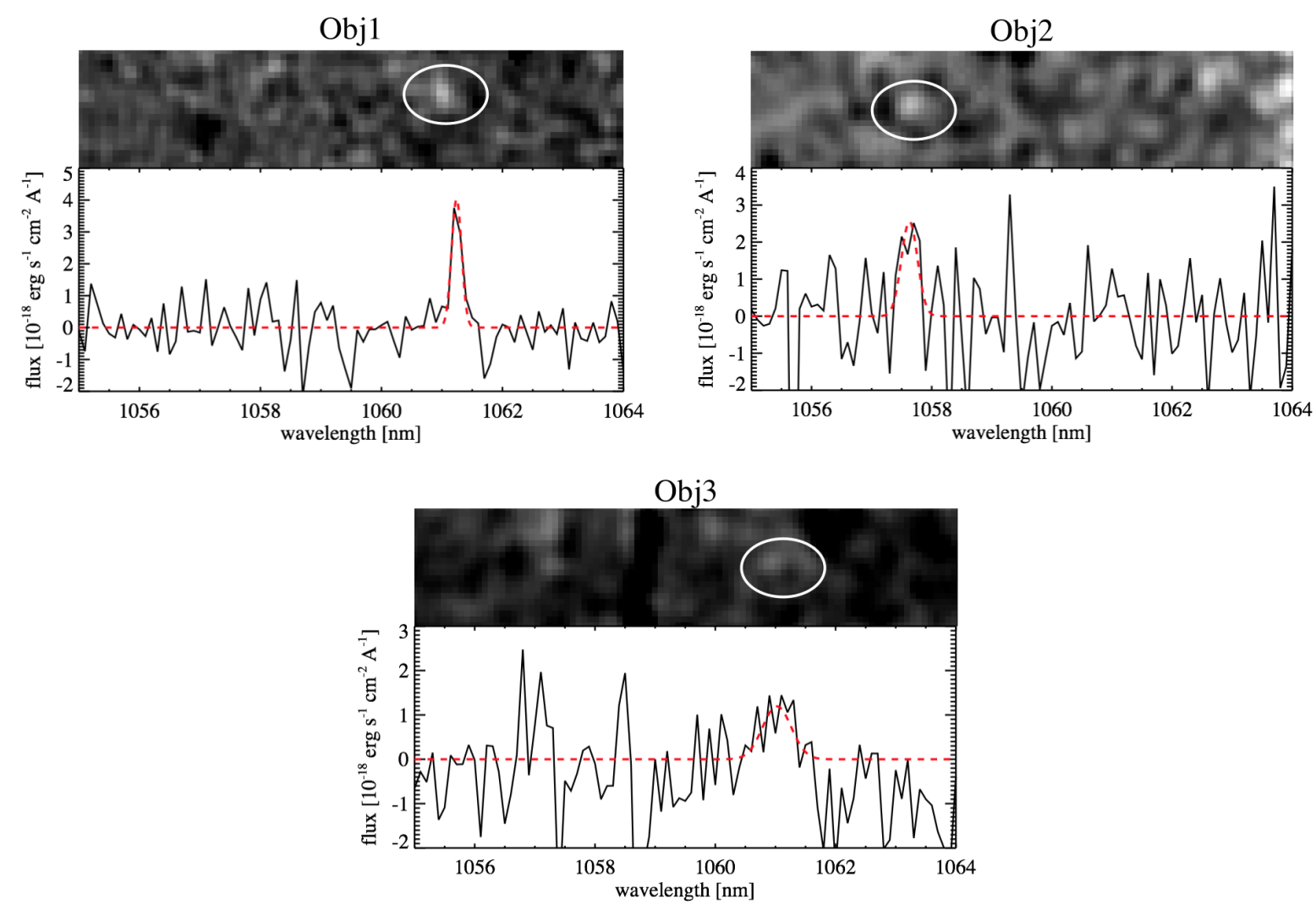

Fig. 2. 2D and 1D spectra centred on the line detected in NB1060 for each object. The red dashed line is the Gaussian fit of the line.

the slit width, and the sizes of each object as measured on the NB images. The accuracy of the estimate of the slit losses is low, and is estimated to be close to $30 \%$. This introduces systematic uncertainties in the scientific analysis that follows, however, without altering our main conclusions. We estimated the level of the noise on the 1D spectra by measuring the standard deviation on both sides of the line on $\sim 30$ pixels (equivalent to $\sim 3 \AA$ ).

\subsection{Methodology and application to Object 1}

The central wavelength of the line is $\lambda=1061.3 \mathrm{~nm}$. Fitting a Gaussian profile to the line, and after applying a correcting factor of 2 to account for slit losses, we measure an integrated flux of $1.50 \times 10^{-17} \mathrm{erg} \mathrm{s}^{-1} \mathrm{~cm}^{-2}$ with a $\mathrm{S} / \mathrm{N}$ of 7.7 .

The measured spectroscopic line flux is equivalent to a magnitude of 24.55 over the bandwith of the NB filter, indicating that the line flux indeed dominates the NB1060 magnitude of the object (24.3). We derive a continuum level of $\sim 3.93 \times$ $10^{-20} \mathrm{erg} \mathrm{s}^{-1} \mathrm{~cm}^{-2} \AA^{-1}$, corresponding to a magnitude of 26.0. This continuum level is far too faint to be detected on the $\mathrm{X}$-Shooter data, and is also consistent with the non-detection in the $J$-band at the $5 \sigma$ limit of our observations, $(A B \sim 26)$. From this continuum level, we also derive an EW of the line of $\sim 400 \AA$. The absolute flux measurements are associated with significant uncertainties (because of the errors on slit losses for instance), leading in turn to large uncertainties on the estimate of the EW.

The detection of a single line prevents a straightforward identification of the redshift of the source. For the purpose of determining this redshift from the whole photometric and spectroscopic data set, we resort to a spectral energy distribution (SED) fitting analysis using LePhare (Arnouts et al. 1999; Ilbert et al. 2006). This code uses a $\chi^{2}$ template fitting method, and for the purpose of our analysis we use spectral templates of dusty starburst galaxies with emission lines.

We used templates from Bruzual \& Charlot (2003) first. These were subsequently modified by LePhare with variable extinction laws (Calzetti et al. 1994) and the addition of emission lines following classical recipes that relate galaxy star formation rates and luminosities in the UV continuum, recombination lines, and forbidden lines (Kennicutt 1998). Moreover, LePhare was run by constraining the redshift to discrete intervals for which one of the [OII], [OIII]4959 $\AA$, [OIII]5007 $\AA$, $\mathrm{H} \beta$, and $\mathrm{H} \alpha$ lines corresponds to the detected emission line. Best fits are shown in Fig. 3 with their associated $\chi^{2}$ values. Overall, LePhare found no good fit, including the NB flux, at any redshift by changing the extinction and the emission lines.

We therefore investigate extreme spectra with high EW emission lines. We use the spectrum of an ultra-strong emission line object (USEL, Kakazu et al. 2007; Hu et al. 2009). USELs are characterized by strong emission lines that can have extreme rest-frame EWs of several hundred $\AA$. We therefore use a representative USEL spectrum to evaluate whether their spectral properties fit our data. We run LePhare first, similar to the starburst case. The results of the fits and of the $\chi^{2}$ values are shown in panel (a) of Fig. 4. The used USEL spectrum, representative of the USEL population, provides a much better fit to the observed photometric data of the object than a dusty starburst spectrum coming from the library of Bruzual \& Charlot (2003) if the detected line is [OIII](5007) $\AA$. Indeed, the reduced $\chi^{2} \sim 1$ as compared to several hundreds. 


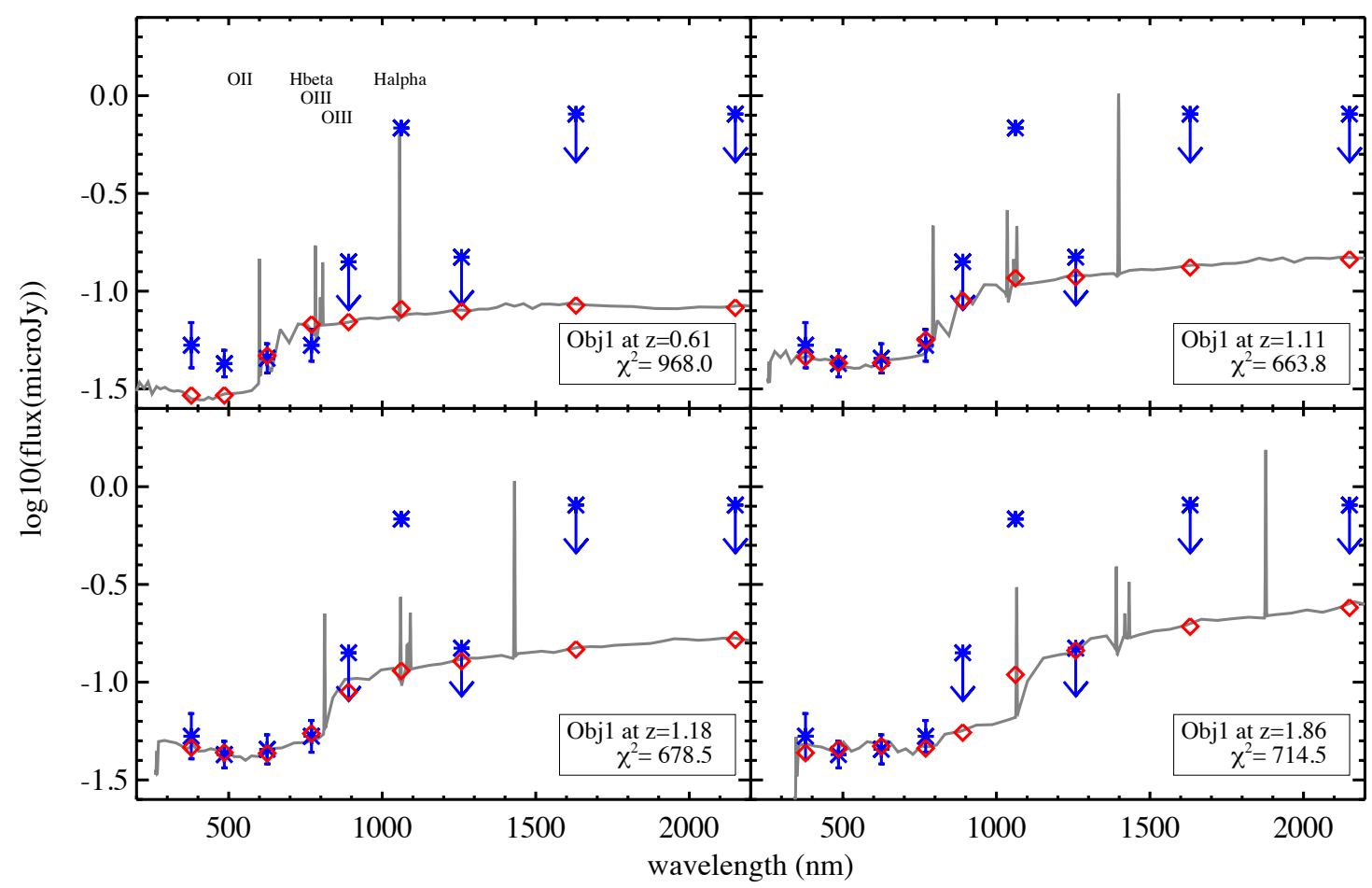

Fig. 3. Results of the SED fitting with LePhare for Obj1 using Bruzual \& Charlot (2003) models and assuming that the detected line is H $\alpha$ at $z \sim 0.61$, [OIII] at $z \sim 1.11, \mathrm{H} \beta$ at $z=1.18$, and [OII] at $z \sim 1.86$. The grey line is the best-fit SED. Blue crosses are measured magnitudes or magnitude upper limits while red squares are the expected magnitudes coming from the fit.

We then investigate whether other emission lines should have been detected in the X-Shooter data. Here again, we investigate two types of spectra: standard star-forming galaxies with and without extinction (Cassata et al. 2011) and USELs. We assume that the detected line is one of the five [OII], [OIII]4959 $\AA$, [OIII]5007 $\AA, \mathrm{H} \beta$, and $\mathrm{H} \alpha$ emission lines, and we compute in turn the $\mathrm{S} / \mathrm{N}$ expected in our data on the three other lines. The line ratios without extinction are indicated in Table 3. In the case of star-forming galaxies without extinction, it is impossible to reproduce a situation where only one line could be detected. In all situations, at least one line should have been detected with a $\mathrm{S} / \mathrm{N}$ above 2 . However, considering a relatively high level of extinction of $E(B-V)=0.4$, the single detection of the $\mathrm{H} \alpha$ line in the NB filter is possible. Nevertheless, this possibility has been excluded by the LePhare analysis. In the case of USELs, if the measured line is [OIII]5007 $\AA$, no other lines could have been detected in our data ( $\mathrm{S} / \mathrm{Ns}$ between 1 and 2$)$. Indeed, the [OIII]5007 A line strongly dominates the USEL spectra (see Table 3). No coincidence with sky absorption or emission lines could have explained the single line detection in our data in all other situations than this line being [OIII]5007 $\AA$. In conclusion of this section, there is convincing evidence that Obj1 is a USEL [OIII] emitter at $z=1.12$.

\subsection{Object 2}

The central wavelength of the line is $\lambda=1057 \mathrm{~nm}$. Fitting a Gaussian profile and applying the slit loss correction factor of 2 , its integrated flux is $1.71 \times 10^{-17} \mathrm{erg} \mathrm{s}^{-1} \mathrm{~cm}^{-2}$ with a S/N of 5.1. Over the bandwidth of the NB1060 filter the measured spectroscopic flux is equivalent to a magnitude of 24.4 , therefore the line flux dominates the NB1060 magnitude of the object (24.3).
Table 3. The two sets of flux ratios considered in that study.

\begin{tabular}{lcc}
\hline \hline Line ratio & Star-forming & USELs \\
\hline$[\mathrm{OIII}] 5007 /[\mathrm{OIII}] 4959$ & 3 & 3 \\
{$[\mathrm{OIII}] 5007 /[\mathrm{OII}]$} & 0.35 & 4.5 \\
$\mathrm{H} \alpha /[\mathrm{OII}]$ & 1 & 2.5 \\
$\mathrm{H} \beta / \mathrm{H} \alpha$ & 0.35 & 0.35 \\
\hline
\end{tabular}

Notes. Those for star-forming galaxies come from Cassata et al. (2011) and those for USELs are from Kakazu et al. (2007) and Hu et al. (2009).

The derived continuum level is $\sim 1.8 \times 10^{-20} \mathrm{erg} \mathrm{s}^{-1} \mathrm{~cm}^{-2} \AA^{-1}$ corresponding to a magnitude of 26.8 , which is again consistent with the non-detection in the $J$-band at $5 \sigma$. Using that level of continuum we derive an EW of $\sim 1000 \AA$.

LePhare does not allow us to find a suitable fit solution with Bruzual \& Charlot (2003) templates whereas the USEL template provides a good fit to the observed photometric data as shown in panel (b) of Fig. 4 (reduced $\chi^{2} \sim 2$ as compared to several hundreds). The check for the detection of other emission lines leads to either the strong [OIII] $5007 \AA$ line of a USEL or the $\mathrm{H} \alpha$ line of an extinct star-forming galaxy, the latter being inconsistent with the LePhare results. In conclusion, Obj2 is also a USEL [OIII] emitter at $z=1.12$ and the EW of the [OIII] line from the USEL template is $830 \AA$, consistent, within error bars, with our observations.

\subsection{Object 3}

One single emission line is detected in the 2D spectrum with a central wavelength of $\lambda=1061 \mathrm{~nm}$ (see Fig. 2). The fit of a Gaussian profile to the line and the application of a correcting 


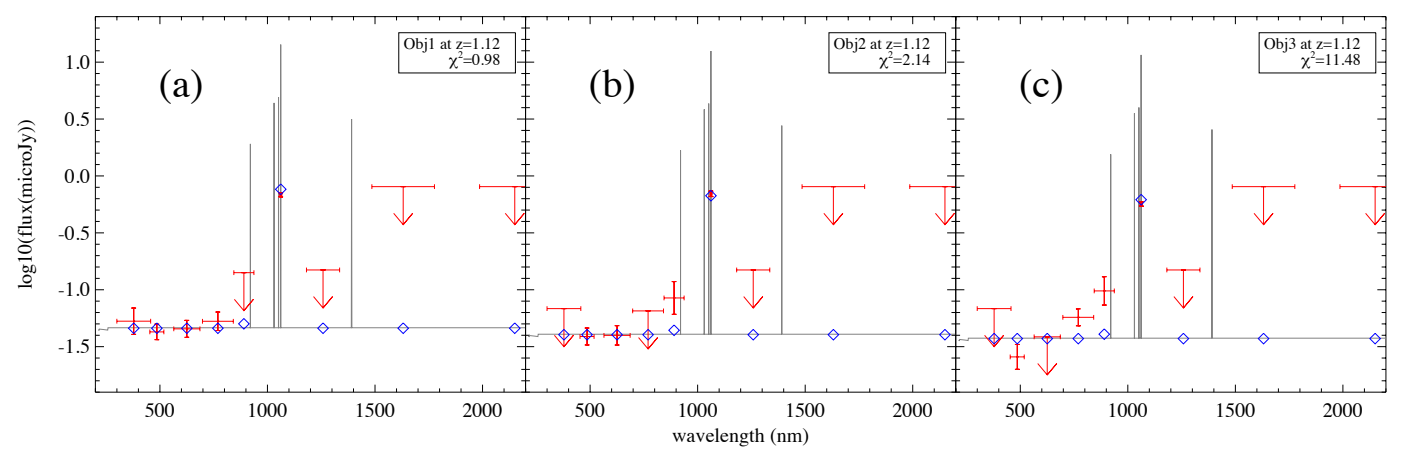

Fig. 4. Fits of the three objects magnitudes using LePhare with a USEL template assuming that the detected line is [OIII]5007 A. Red crosses are the measured magnitudes while arrows are upper limits. The blue diamonds show the magnitudes predicted by LePhare and the grey line is the best-fit spectrum from LePhare.

factor of 1.7 to account for slit loss lead to a measured integrated flux of $1.27 \times 10^{-17} \mathrm{erg} \mathrm{s}^{-1} \mathrm{~cm}^{-2}$ with a $\mathrm{S} / \mathrm{N}$ of 3.2. This line flux is equivalent to a magnitude of 24.7 over the bandwidth of the $N B 1060$ filter, the $N B 1060$ magnitude is therefore dominated by the line flux of the object (24.52). The estimated continuum level is $\sim 3.0 \times 10^{-20} \mathrm{erg} \mathrm{s}^{-1} \mathrm{~cm}^{-2} \AA^{-1}$ corresponding to a magnitude of 26.3. Similar to Obj1, the continuum is too faint to be detected in the X-Shooter data and is consistent with the non-detection in the $J$-band $(A B \sim 26$ at $5 \sigma)$. The EW of the line associated with this continuum is $\sim 420 \AA$.

We carried out the same analysis using LePhare as for Obj1, and found no good fit solution from the templates of Bruzual $\&$ Charlot (2003) (reduced $\chi^{2}$ of several hundreds). We checked whether other emission lines could have been detected in the cases of standard star-forming galaxies (with and without extinction) and USELs. In the former case, the detected line could be $\mathrm{H} \alpha$ as the $\mathrm{S} / \mathrm{Ns}$ of the other lines are lower than $2 \sigma$. However, this possibility is ruled out by the LePhare analysis. Indeed, the magnitude in the NB1060 filter predicted by the template fitting is strongly inconsistent with the observed magnitude (2 mag difference). Considering USELs, only the detection of the [OIII] line is consistent with no detection of other lines in our data. However the LePhare fit is not satisfying either as the reduced $\chi^{2}$ is $\sim 11$, as shown in panel (c) of Fig. 4 . In conclusion, Obj3 is likely to be a USEL [OIII] emitter at $z=1.12$. The [OIII] EW measured from the USEL template is $\sim 670 \AA$, which is consistent within the error bars with our observations.

\section{Discussion and implication for high-z surveys}

We followed three peculiar objects selected as high-EW line emitters spectroscopically in a search for LAE candidates at $z \sim 7.7$ using very deep optical data (Clément et al. 2012). Had we used optical data that was shallower by approximately one magnitude, we would have selected these three objects as genuine LAE candidates at $z \sim 7.7$. These objects have magnitude differences ranging from 2.3 to 3.5 between the five optical (ugriz) bands and the NB1060 band. This represents large colour breaks, considering that the optical-NB breaks used for selecting LAE samples at high redshift is of the order of 1.5 to $2.5 \mathrm{mag}$ in most studies (for instance, Hibon et al. 2012; Shibuya et al. 2012; Konno et al. 2014). Indeed, from Table 1, we infer that with the use of colour breaks of one magnitude lower, the three objects would have been selected as LAE candidates. Furthermore, these objects could also contaminate high- $z$ LBG samples through broadband detection of their strong line emission. In a thorough study of LBG selection criteria, Capak et al. (2011) remarked that USELs are a potential source of interlopers in $z>7$ galaxy searches and conclude that the $i$-band data must be 4 mag deeper than the $J$-band data.

These three objects are therefore potential contaminants to high- $z$ galaxy samples and unveiling their nature is of interest for estimating the contamination rate of these samples. Despite the low quality of our data because of poor observing conditions during the observations, we identify emission lines in spectroscopy at the position corresponding to the wavelength range of the NB1060 filter. We do not find other emission lines in the X-Shooter data for any of these objects. After SED fitting and detailed analysis of the spectroscopic data, we argue that the emission line in the NB1060 filter is [OIII]5007 $\AA$, placing these three contaminants at a redshift of $\sim 1.1$.

This claim is consistent with similar reports of spectroscopically confirmed contaminants available in the literature that highlight the importance of [OIII] emission. These objects are also referred to as extreme emission line galaxies (Huang et al. 2015, for instance). In searching LBGs at redshift between 8 and 10 from deep near IR imaging, Richard et al. (2003) identified a [OIII] emitter at $z=1.68$ from spectroscopic follow-up observations). This object is characterized by a high EW and it has $[\mathrm{OIII}] 5007 / \mathrm{H} \beta \sim 5.9$ fully consistent with USEL values. Similarly, Hayes et al. (2012) followed a $z \sim 11$ LBG candidate with $\mathrm{X}$-Shooter and detected several emission lines, amongst which [OIII] is the strongest with an EW of $\sim 700 \AA$, which put that galaxy at $z=2.08$. They conjecture that this object is either a heavily obscured starburst or an old galaxy upon which a burst of star formation is superimposed. The example of UDFj-39546284 is illustrative of the extreme difficulty of confirming very high-redshift galaxy candidates and of the role of faint low $z$ interlopers. This object was originally claimed to be at $z \sim 10$ (Bouwens et al. 2011). With deeper near IR data, Ellis et al. (2013) suggested that this object could lie at an even higher redshift of $z=11.9$. From HST WFC3 grism observations Brammer et al. (2013) detected a $2.7 \sigma$ line that could either be Ly- $\alpha$ at $z=12.12$ or [OIII] at $z=2.19$, Bouwens et al. (2013) finding the later solution more plausible. From Keck-MOSFIRE data, Capak et al. (2013) could not improve the line detection level and therefore reach a conclusion on the nature of this object. All these examples suggest the prevalence of [OIII] emitters amongst interlopers to high- $z$ galaxy candidates is in line with our findings.

Finally, as a last sanity check, we estimate the number of strong [OIII] emitters expected in the CFHTLS D4 field from 
their luminosity function at redshift 0.8 as reported in Fig. 13 of Kakazu et al. (2007). We assume no evolution in redshift between 0.8 and 1.1, and we further assume that the NB1060 flux is entirely dominated by [OIII] emission. The redshift interval probed by the $N B 1060$ filter is 0.02 for the [OIII] line, corresponding in turn to a co-moving volume of $\sim 3 \times 10^{3} \mathrm{Mpc}^{3}$ for our CFHTLS D4 field. We consider a NB1060 limiting magnitude of 24.6, corresponding to a line luminosity of $10^{41} \mathrm{erg} \mathrm{s}^{-1}$. We derive that there should be approximately four ultra-strong [OIII] emitters in our data at the flux limit that we consider, in good agreement, within low number statistics and large uncertainties, with the three objects that we selected. It is reassuring that both numbers match, comforting the likelihood that the three objects are indeed [OIII] emitters. There were seven objects in the CFHTLS-D4 field displaying similar photometric properties, and the other four objects have NB1060 magnitudes fainter than 24.6 .

We infer from this study that [OIII] emitting objects are a likely important source of contamination in NB-selected highredshift galaxy samples, and preventing contamination from these objects require optical data deeper than the NB data by almost 3 mag. As noted above, Capak et al. (2011) suggest 4 mag difference to prevent $z>7$ galaxy (LBG) samples from being contaminated by low- $z$ interlopers.

Interestingly, a majority of the $z \sim 2.2 \mathrm{H} \alpha$ emitter candidates originally selected from NB imaging at $2.2 \mu \mathrm{m}$ in Moorwood et al. (2000) turned out to be [OIII] emitters after detailed followup analyses (Moorwood et al. 2003). Matthee et al. (2014) and Kochiashvili et al. (2015) recently performed photometric redshift analyses of NB emitters at $1.18 \mu \mathrm{m}$ and $1.06 \mu \mathrm{m}$ respectively, and they find a fraction of $\mathrm{H} \beta /[\mathrm{OIII}]$ emitters of $40 \%$ and $28 \%$ respectively. Combined to an analysis of the broadband photometric data, these analyses could inform us further on the likelihood of strong [OIII] emitters in NB studies.

The occurrence of strong [OIII] emitters seems to be ubiquitous over a large redshift range, and such objects are a likely source of contamination/error in many studies. A more detailed analysis of the [OIII] luminosity functions at redshifts up to 3 is needed to further evaluate the level of contamination that [OIII] might generate in several of the planned dark energy surveys. Strong [OIII] emission might contaminate photometric redshifts, be they directly used for scientific analyses (e.g. Euclid) or for photometric selection of targets to be subsequently followedup in spectroscopy (e.g. PFS/SuMIRe or DESI). We suggest that the [OIII] luminosity function should be better determined over a large redshift range, as recently completed by Comparat et al. (2015) for the [OII] luminosity function over the redshift range [0.1-1.65]. This kind of study can be carried out using the WISP (WFC3 Infrared Spectroscopic Parallel) survey (Atek et al. 2010, PI: Malkan) in which several hundred USELS were found by Atek et al. (2014) over the redshift range $0.3<z<2.3$. Even if it is crucial, the characterization of the [OIII] luminosity function at higher redshift will be possible only with IR space telescopes such as the JWST. Only the detailed knowledge of the [OIII] luminosity function could allow us to better estimate the contamination rate of any high- $z$ galaxy survey from [OIII] emitters.
Acknowledgements. The authors would like to thank the referee whose comments improved this manuscript. We thank Hakim Atek, Brian Lemaux, Danka Paraficz, Johan Richard, and Tayyaba Zafar for valuable discussions. We thank Len Cowie for providing the USEL templates. This work received support from the Agence Nationale de la Recherche bearing the reference ANR-09-BLAN0234. J.P.K. acknowledges support from ERC advanced LIDA and from CNRS.

\section{References}

Arnouts, S., Cristiani, S., Moscardini, L., et al. 1999, MNRAS, 310, 540 Atek, H., Malkan, M., McCarthy, P., et al. 2010, ApJ, 723, 104 Atek, H., Siana, B., Scarlata, C., et al. 2011, ApJ, 743, 121 Atek, H., Kneib, J.-P., Pacifici, C., et al. 2014, ApJ, 789, 96 Bouwens, R. J., Illingworth, G. D., Labbe, I., et al. 2011, Nature, 469, 504 Bouwens, R. J., Oesch, P. A., Illingworth, G. D., et al. 2013, ApJ, 765, L16 Bouwens, R. J., Illingworth, G. D., Oesch, P. A., et al. 2015, ApJ, 803, 34 Brammer, G. B., van Dokkum, P. G., Illingworth, G. D., et al. 2013, ApJ, 765, L2

Bruzual, G. \& Charlot, S. 2003, MNRAS, 344, 1000

Calzetti, D., Kinney, A. L., \& Storchi-Bergmann, T. 1994, ApJ, 429, 582

Capak, P., Mobasher, B., Scoville, N. Z., et al. 2011, ApJ, 730, 68

Capak, P., Faisst, A., Vieira, J. D., et al. 2013, ApJ, 773, L14

Cassata, P., Le Fèvre, O., Garilli, B., et al. 2011, A\&A, 525, A143

Clément, B., Cuby, J.-G., Courbin, F., et al. 2012, A\&A, 538, A66

Comparat, J., Richard, J., Kneib, J.-P., et al. 2015, A\&A, 575, A40

Cuby, J.-G., Hibon, P., Lidman, C., et al. 2007, A\&A, 461, 911

D'Odorico, S., Dekker, H., Mazzoleni, R., et al. 2006, in SPIE Conf. Ser., 6269

Ellis, R. S., McLure, R. J., Dunlop, J. S., et al. 2013, ApJ, 763, L7

Finkelstein, S. L., Papovich, C., Dickinson, M., et al. 2013, Nature, 502, 524

Hayes, M., Laporte, N., Pelló, R., Schaerer, D., \& Le Borgne, J.-F. 2012, MNRAS, 425, L19

Hibon, P., Cuby, J.-G., Willis, J., et al. 2010, A\&A, 515, A97

Hibon, P., Malhotra, S., Rhoads, J., \& Willott, C. 2011, ApJ, 741, 101

Hibon, P., Kashikawa, N., Willott, C., Iye, M., \& Shibuya, T. 2012, ApJ, 744, 89

Hu, E. M., Cowie, L. L., Capak, P., et al. 2004, AJ, 127, 563

Hu, E. M., Cowie, L. L., Kakazu, Y., \& Barger, A. J. 2009, ApJ, 698, 2014

Hu, E. M., Cowie, L. L., Barger, A. J., et al. 2010, ApJ, 725, 394

Huang, X., Zheng, W., Wang, J., et al. 2015, ApJ, 801, 12

Ilbert, O., Arnouts, S., McCracken, H. J., et al. 2006, A\&A, 457, 841

Kakazu, Y., Cowie, L. L., \& Hu, E. M. 2007, ApJ, 668, 853

Kashikawa, N., Shimasaku, K., Malkan, M. A., et al. 2006, ApJ, 648, 7

Kennicutt, Jr., R. C. 1998, ARA\&A, 36, 189

Kochiashvili, I., Møller, P., Milvang-Jensen, B., et al. 2015, A\&A, accepted, ArXiv e-prints [arXiv: 1504.3005]

Konno, A., Ouchi, M., Ono, Y., et al. 2014, ApJ, 797, 16

Krug, H. B., Veilleux, S., Tilvi, V., et al. 2012, ApJ, 745, 122

Matthee, J. J. A., Sobral, D., Swinbank, A. M., et al. 2014, MNRAS, 440, 2375

Milvang-Jensen, B., Freudling, W., Zabl, J., et al. 2013, A\&A, 560, A94

Moorwood, A. F. M., van der Werf, P. P., Cuby, J. G., \& Oliva, E. 2000, A\&A, 362,9

Moorwood, A., van der Werf, P., Cuby, J. G., \& Oliva, T. 2003, in The Mass of Galaxies at Low and High Redshift, eds. R. Bender, \& A. Renzini, 302

Nakamura, E., Inoue, A. K., Hayashino, T., et al. 2011, MNRAS, 412, 2579

Ouchi, M., Shimasaku, K., Furusawa, H., et al. 2010, ApJ, 723, 869

Pentericci, L., Vanzella, E., Fontana, A., et al. 2014, ApJ, 793, 113

Richard, J., Schaerer, D., Pelló, R., Le Borgne, J.-F., \& Kneib, J.-P. 2003, A\&A, 412, L57

Schenker, M. A., Stark, D. P., Ellis, R. S., et al. 2012, ApJ, 744, 179

Shibuya, T., Kashikawa, N., Ota, K., et al. 2012, ApJ, 752, 114

Steidel, C. C., Adelberger, K. L., Shapley, A. E., et al. 2003, ApJ, 592, 728

Tilvi, V., Rhoads, J. E., Hibon, P., et al. 2010, ApJ, 721, 1853

Tilvi, V., Papovich, C., Finkelstein, S. L., et al. 2014, ApJ, 794, 5

Trenti, M., Bradley, L. D., Stiavelli, M., et al. 2011, ApJ, 727, L39

Vanzella, E., Pentericci, L., Fontana, A., et al. 2011, ApJ, 730, L35

Vernet, J., Dekker, H., D’Odorico, S., et al. 2011, A\&A, 536, A105

Willis, J. P., Courbin, F., Kneib, J.-P., \& Minniti, D. 2008, MNRAS, 384, 1039 